\title{
Geschlechterdualismen in der Wohlfahrtspflege: "Soziale Mütterlichkeit" zwischen Professionalisierung und Medikalisierung, Deutschland 1890-1930
}

Gabriele Czarnowski, Elisabeth MeyerRenschhausen

In Berlin wurde die Fachhochschule für Sozialarbeit und Sozialpädagogik, die heutige Alice-Salomon-Schule, in den Topf der großen Umschichtungsmaßnahmen gesteckt. Vom angestammten Platz in Schöneberg, einem Bezirk im Herzen der Hauptstadt, mit zunehmenden Verslumungsprozessen, wo ausländische Jugendliche keine Ausbildungsplätze erhalten und so dem Drogenhandel in die Arme getrieben werden, wo Prostitution, Obdachlose, Alkoholiker/innen und elende Alte aus dem Straßenbild nicht mehr wegzudenken sind, soll die Schule an den äußersten Stadtrand Berlins nach Hellersdorf verlegt werden. Hellersdorf ist eine moderne Trabantenstadt mit 250.000 Einwohner/inne/n mit einem Durchschnittsalter von 30 Jahren. Seit der Wende mehrt sich hier die Arbeitslosigkeit von jungen Frauen. Die Alice-Salomon-Schule soll verlegt werden, um jungen Frauen im mit Ausbildungsmöglichkeiten unterversorgten Ostberlin Qualifizierungsmöglichkeiten zu verschaffen. Sozialarbeit wird als Ausbildungsberuf und Arbeitsmöglichkeit behandelt.

Als die ersten Vorläufer der späteren Schulen für Sozialarbeit von der ersten Frauenbewegung vor gut 100 Jahren 1893 gegründet wurden, ging es keineswegs um Ausbildungsplätze, wohl aber darum, junge Frauen zum Beheben sozialer Ungerechtigkeiten zu qualifizieren. Die - emphatisch so genannte - „soziale Arbeit" wurde ais frauenspezifischer Beitrag zu einer notwendigen Gesellschaftsreform verstanden. $\mathrm{Zu}$ einem Ausbildungsberuf, der Frauen notwendige Erwerbsarbeitsplätze verschaffte, wurde die Sozialarbeit erst in den 1920 er Jahren. Die Gründerinnen-Generation war über diese Entwicklung nicht froh. Sie fürchtete, daß die "soziale Arbeit" so um ihre gesellschaftliche Mission gebracht werden könnte, die Einseitigkeiten und Ungerechtigkeiten der damaligen "Männergesellschaft" abzuschaffen. Statt alles allein in Geld und Wirtschaftsprozessen zu rechnen, soliten auch die "menschlichen" Seiten des Lebens, d. h. die Menschen in ihren natürlichen und ästhetischen Bedürfnissen, $\mathrm{Be}$ - 
achtung finden. Die Sozialreformerinnen des frühen 20. Jahrhunderts strebten eine Gesellschaft an, in der Haus- und Handarbeiten ebenso geachtet würden wie Organisations- und Denkarbeiten. Hannah Arendt hat uns klar gemacht, daß die Frauenrechtlerinnen der Jahrhundertwende sich damit gegen männliche Weltsichten wandten, die seit den Zeiten der griechischen klassischen Antike körperliche Arbeiten im Hause wie außerhalb desselben als niedrige Tätigkeiten werteten. Seit Adam Smiths Zeiten huldigte dieses Denken einem atomisierten homo oeconomicus als ausschließlichem Denkmodell. ${ }^{1}$

Die Weltsicht, die den Menschen vom Prinzip des Abstrakten her dachte, nämlich vom jeweils „übergeordneten" gesellschaftlichen Organisationsmodell, statt den Menschen vom Konkreten her, in seinen jeweiligen Gruppenbezügen zu denken, blieb vielen Frauen fremd. ${ }^{2}$ Die Aufklärer hatten den Frauen zugestanden, der von ihnen angestrebte allseitig gebildete neue Mensch zu werden, als sie bereits Ende des 18. Jahrhunderts erkannten, daß der männliche Bürger sich zugunsten seiner beruflichen Laufbahn ins Korsett des einseitigen „Experten" zwingen lassen mußte. ${ }^{3}$ Die Frauen, die die Gesellschaft "aus dem Haus", von der Familie her dachten, hatten sich daher auch im 19. Jahrhundert das ,Sich-für-den-Nächsten-verantwortlichFühlen" noch nicht abgewöhnen müssen. ${ }^{4}$ Sie standen in ihrem Denken damit in der Tradition der Gruppengesellschaften des alten Europa, einem Denken in der Tradition einer "moral economy". 5

\section{Von der Armenfürsorge zu Sozialversicherung und Sozialer Frauenarbeit}

In der Alten Gesellschaft galten wie im ländlichen Europa noch bis ins 20. Jahrhundert hinein Systeme gegenseitiger Hilfe. Wenn es die Not erforderte, halfen Nachbar/inne/n Nachbar/inne/n. Familienangehörige Familienangehörigen und Zunftgenossen Zunftgenossen, auch unabhängig von geschriebenem Recht. Diese alten "privaten" Hilfssysteme reichten erst dann nicht mehr aus, als seit dem ausgehenden Mittelalter ökonomische Umschichtungen der europäischen Gesellschaften begannen. Immer mehr Menschen wurden um "Haus und Hof" gebracht, "auf die Straße" geworfen und strömten als mittellose Migrant/inn/en und Bettler/innen in die Städte. Diese neue Gruppe Hilfsbedürftiger, Fremde in einer für sie neuen Kultur, konnten auf die spontanen Zuwendungen überschaubarer Gemeinschaften nicht

1 Hannah Arendt, Vita activa, München ${ }^{4} 1985$.

2 Dieter Claessens, Das Konkrete und das Abstrakte, Frankfurt a. M. 1980.

3 Barbara Duden, Die Berliner Salons um 1800. Zur Emanzipation der Frauen. Hausarb. Berlin 1970.

4 Elisabeth Meyer-Renschhausen, Weibliche Kultur und soziale Arbeit. Eine Geschichte der Frauenbewegung 1810-1927 am Beispiel Bremens, KölnMien 1989.

5 Edward P. Thompson, Die „sittliche Okonomie“ der englischen Unterschichten im 18. Jahrhundert, in: Detlev Puls Hg., Wahrnehmungsformen und Protestverhalten. Studien zur Lage der Unterschichten im 18. und 19. Jahrhundert. Frankfurt a. M. 1979, 13-88. 
mehr bauen. Die Städte sahen sich gezwungen, regelmäßige Armensteuern einzuheben und Satzungen zu entwerfen, die den Umgang mit in Not Geratenen regelten. Das erste Arbeits- und Zuchthaus entstand 1604 in Amsterdam. Soziale Disziplinierung war seither Ziel staatlicher und kommunaler Armenfürsorge. Seit dem 17. Jahrhundert wurden in den größeren Städten Armenordnungen schriftlich niedergelegt. Der absolutistische Staat verfestigte die Zuständigkeit der Kommunen für mittellose Untertanen. Im Gegensatz zur alten nachbarschaftlichen Hilfe machte ein von Staats wegen von oben nach unten gedachtes Armenversorgungssystem plötzlich nötig, zu definieren, wer als Hilfsbedürftige/r gelten sollte.

Mit dem Unterstützungswohnsitzgesetz von 1870 machte der entstehende Zentralstaat den Kommunen diese Form der Fürsorge zur Pflicht, ohne jedoch seinerseits Verpflichtungen zu übernehmen. ${ }^{6}$ Auch nach der Reichsgründung 1871 war die Art der Versorgung der Unbemittelten uneinheitlich, weil sie kostenmäßig Angelegenheit der Städte und Landgemeinden blieb. In den 1880er Jahren übernahm das Reich durch die gesetzliche Einführung der Arbeiterversicherungen einen Teil der sozialen Fürsorge, die für Kranke (1883), für Opfer von Arbeitsunfällen (1884) und schließlich Invalide und Alte (1889) nach und nach gewisse Erleichterungen brachte. Diese Zwangsversicherungen - Arbeiter/innen und Arbeitgeber/innen wurden vom Staat verpflichtet, in entsprechende Kassen einzuzahlen - führten zu jenem Sozialversicherungssystem, das in den 1920er Jahren weltweit als das umfassendste galt. Die Sozialversicherungen traten neben die Bismarcksche Politik der politischen Unterdrückung der Arbeiterbewegung, die im "Gesetz gegen die gemeingefährlichen Bestrebungen der Sozialdemokratie" (1878-1890) verankert war.

Kennzeichnend für die neuen Arbeiterversicherungssysteme war, daß sie unmittelbar an den Lohnarbeiterstatus anknüpften. Sie galten nur für regelmäßige „Vollzeit"-Lohnarbeiter. ${ }^{7}$ Zugespitzt kann man sagen, daß diese neuen Absicherungssysteme den modernen regulären Lohnarbeiter erst schufen, indem sie ihn auf diese Arbeitsweise verpflichteten. Ständig Beschäftigten wurde durch dieses System nun garantiert, daß sie im Fall von Krankheit oder Invalidität die degradierende Armenhilfe nicht mehr in Anspruch zu nehmen brauchten. Sie gehörten wieder zu den "ehrbaren Leuten". 8 Zugleich wurden durch das Sozialversicherungssystem die Unterschiede innerhalb der - wie es in der Sprache der Zeit hieß - „Klasse der Besitzlosen“ verschärft. Denn für alle „unständig" Beschäftigten, wie Hafenarbeiter, Tagelöhner/innen, Gelegenheitsarbeiter/innen aller Art und die in Haus und

6 Vgl. u. a. Eberhard Orthbandt, Der deutsche Verein in der Geschichte der Deutschen Fürsorge (= Schriften des Deutschen Vereins für öffentliche und private Fürsorge, 260), Frankfurt a. M. 1980

7 Vgl. dazu Christoph Sachße. Mütterlichkeit als Beruf. Sozialarbeit, Sozialreform und Frauenbewegung 1871-1929. Frankfurt a. M. 1986; Orthbandt. Verein, wie Anm. 6. Eine allgemeine staatliche Arbeitslosenversicherung wurde erst 1927 eingeführt

8 Vgl. Heide Gerstenberger, Von der Armenpflege zur Sozialpolitik, in: Leviathan, 9. 1 (1981), $57 f$. 
Landwirtschaft abhängig Arbeitenden wie Dienstmädchen und Heimarbeiter/innen galt die Arbeiterversicherung nicht. ${ }^{9}$ Im Falle von Krankheit oder Invalidität fielen sie weiterhin den örtlichen Armenpflegesystemen mit ihren diskriminierenden Wirkungen „anheim".

Das neue Sozialversicherungssystem führte zur weiteren gesellschaftlichen Ausbreitung des modernen Geschlechtergegensatzes. Für Frauen wirkten sich die Sozialversicherungen eher negativ aus. Sie gehörten in ihrer Mehrzahl nicht zur Gruppe der regelmäßig beschäftigten Lohnarbeiter/innen. Wenn sie jünger waren, arbeiteten sie als Magd, Dienstbotin oder Fabrikarbeiterin, wenn sie Mütter und älter waren, als Heimarbeiterin, "Grünkrämerin", Tagelöhnerin, als Büglerin, Wäscherin, Zugehfrau, Näherin oder "Privatlehrerin". Auf dem Lande - hier lebte um 1900 noch die Hälfte der Bevölkerung betrieben meist die Frauen die Nebenerwerbslandwirtschaft und trugen damit erheblich zum Familieneinkommen bei. Der Bauernhof, ihre „häusliche Wirtschaft", zahlreiche andere „Familiengeschäfte" machten hier reguläre Vollzeitlohnarbeit für viele Frauen unmöglich. Die Zahl der eigentlichen Fabrikarbeiterinnen blieb bis in die 1920er Jahre vergleichsweise niedrig. ${ }^{10}$

Die Sozialversicherung diskriminierte die weiblich dominierten Mischwirtschaftssysteme aus Haus-, Feld-, Garten- und diversen Lohnarbeiten. Auf Familienalltag und Familienbedürfnisse nahmen die Versicherungsplaner keine Rücksichten. In manchen Regionen wie in der Oberlausitz protestierten Frauen gegen die Einführung der Sozialversicherungen. Ihnen mißfiel, daß diese ihre Männer auf regelmäßige Arbeit verpflichteten. Die Männer standen innen damit für Noteinsätze in der Nebenerwerbslandwirtschaft wie den Ernteeinsatz und in anderen „weiblichen Arbeitsbereichen“ nicht mehr zur Verfügung."

Die Sozialversicherungen "feminisierten" also die Nebenerwerbslandwirtschaft und alle anderen Hausarbeiten. Sie trugen mit dazu bei, die Ehemänner der besitzlosen Klassen auf das bürgerliche Familienmodell mit dem Mann als "Familienernährer" auszurichten. Frauen wurden von ihren Männern materiell abhängig und konnten als Witwen und Eheverlassene, ledige Mütter und Tagelöhnerinnen leicht zur Klientel der örtlichen Armenversorgung werden.

Damit war der "vierte Stand" der Besitzlosen aufgespalten in eine Klasse der abgesicherten Lohnarbeiter/innen und eine Unterklasse städtischer und ländlicher „Elendsschichten“. Mit der schrittweisen Ausweitung der Sozialversichungen auch auf andere Gruppen männlicher Arbeiter kurz vor dem Ersten Weltkrieg bestand dieses neue

9 Lediglich die Krankenversicherung galt von Anfang an für zwei Drittel der „gewerblichen Arbeiter", nicht jedoch für ihre Frauen und Kinder. 1885 waren $10 \%$ der Bevölkerung ( 4,7 Millionen Menschen) versichert.

$10 \mathrm{Vgl}$. Hildegard Meister-Trescher, Frauenarbeit und Frauenfrage, in: Handwörterbuch der Staatswissenschaften. IV, Jena ${ }^{4}$ 1927, 302-353, hier 305.

11 Jean Quartaert, Teamarbeit in sächischen Handweberfamilien im 19. Jahrhundert. Eine einleitende Untersuchung zur Frage der Geschlechterrollen in der Arbeit, in: Ruth-Ellen B. Joeres u. Annette Kuhn Hg. Frauen in der Geschichte, IV, Düsseldorf 1985, 304-328. 
"Subproletariat" zunehmend aus Frauen und Kindern. ${ }^{12}$ Noch Mitte der 1920er Jahre waren die Krankenkassen zu Leistungen an Familienangehörige im Krankheitsfall nicht gesetzlich verpflichtet. ${ }^{13}$ Weil die Armenfürsorge nur noch als "Unterstock", als letztes Auffangnetz der sozialen Fürsorge galt, war ihre diskriminierende Wirkung verschärft. ${ }^{14}$ Erst mit der Weimarer Reichsverfassung gingen die Ehrenrechte bei Inanspruchnahme öffentlicher Fürsorgeleistungen nicht mehr verloren.

Die Organisationen der Arbeiterbewegung hatten gegen die Einführung der Sozialversicherungen protestiert, weil sie es zu Recht als völlig unzureichendes System begriffen; aber es war bei mehr oder minder verbalen Protesten im Reichstag und in den regionalen Parlamenten geblieben.

Wegen der vorrangigen Bedeutung ihres politischen Fernziels, das kapitalistische Wirtschaftssystem zu stürzen, kümmerte sich die Vorkriegs-Sozialdemokratie kaum um die Praktiken der Armenfürsorge. Auf diesem Gebiet Reformen einzuklagen, überließ sie den „bürgerlichen Sozialreformern", unter ihnen besonders der Frauenbewegung. ${ }^{15} 1880$ wurde der Deutsche Verein für Armenpflege und Wohlthätigkeit gegründet, in dem sich Sozialreformer verschiedenster Berufe und Vertreter von Kommunen und Gebietskörperschaften organisierten. Sie zielten auf eine effektivere Gestaltung der behördlichen Armenversorgung.

Demgegenüber war der Schwerpunkt in der Arbeit der Frauenbewegung ein anderer: Den Frauen ging es um Hilfe für den einzelnen und seine Familie. Ohne kommunale Fragen dabei aus den Augen zu verlieren, bevorzugten sie die behördenunabhängige „private Wohltätigkeit", die sie „soziale Arbeit" nannten. Damit knüpften sie an die Tradition der privaten Armenpflege an, in der Hilfe anzunehmen weniger entehrend war. Wo der Staat durch "Sozialpolitik" die Risiken einer wachsenden Gruppe männlicher Lohnarbeiter zu verringern suchte, begannen die Frauen aus dem Bürgertum, sich mit „Sozialer Arbeit" zugunsten armer Frauen und ihrer Familien einzusetzen. Ihre Arbeit war darauf ausgerichtet, konkrete Hilfsmaßnahmen und „Strukturverbesserungen" vor Ort und en detail zu leisten. Während ihrer Tätigkeit kamen die „sozialen Arbeiterinnen" zunehmend

121911 wurde auch eine Angestelttenversicherung eingefühtt; da viele Frauen jedoch in „ungeschützten Arbeitsverhältnissen" arbeiteten, blieben sie weiterhin unversichert.

13 Nach der Reichsversicherungsordnung waren Leistungen der Krankenkassen für Familienangehörige fakultativ; sie wurden von den Kassenverbänden unterschiedlich gehandhabt

$14 \mathrm{Vgl}$. Sachße, Mütterlichkeit, wie Anm. 7, 34.

$15 \mathrm{Vgl}$. Christoph Sachße u. Florian Tennstedt, Geschichte der Armenfürsorge in Deutschland. Vom Spätmittelatter bis zum 1. Weltkrieg. Stuttgart 1980. Vgl. Irene Stoehr, Housework and motherhood: debates and policies in the women's movement in Imperial Germany and the Weimar Republic, in: Gisela Bock u. Pat Thane $\mathrm{Hg}$., Maternity and Gender Policies. Women and the Rise of the European Welfare States. 1880s-1950s, London/New York 1991, 213-231; dies., Emanzipation zum Staat? Der Allgemeine Deutsche Frauenverein - Deutscher Staatsbürgerinnenverband (1893-1933), Pfaffenweiler 1990. 
zu der Überzeugung, daß Armut in den meisten Fällen Ergebnis der einseitig auf Erwerb und Gewinn ausgerichteten Männerkultur und insofern „unverschuldet" sei. Daher hätten die Armen ein Recht auf Hilfe. Von nicht wenigen Arbeiterfrauen wurde das auch so verstanden. Im Gegensatz zu ihren Ehemännern, die die Hilfe bürgerlicher Frauen als diskriminierend empfinden konnten, hatten sie ein „traditionelleres" Verständnis von sozialer Hilfe im Sinne der alten „moral economy". Als Gelegenheitsarbeiterinnen entwickelten sie im Gegensatz zu ihren Männern keinen - zugespitzt ausgedrückt - „proletarischen Standesstolz". Zum Kern des Lohnarbeiterproletariats gehörten sie nicht. Viele Arbeiterehefrauen blieben daher länger einer ländlich und religiös geprägten Weltsicht treu, die in Notfällen Hilfe zu empfangen als selbstverständlich ansah. ${ }^{16}$

Seit ihrer Entstehung wurde die organisierte Frauenbewegung aktiv in der Tradition von „privater" "Wohltätigkeit" für "unverschuldet" oder „verschämte" Arme. Als Anfang können Lina Morgensterns Suppenküchen gelten, die sie 1866 in Berlin gründete. ${ }^{17}$ Bereits 1868 forderte die Frauenbewegung die Ergänzung des Systems der "Gemeindeväter" durch gleichberechtigte Mitsprach der "Mütter der Gemeinden". Anknüpfend an die Menschenbilddebatte des 18. Jahrhunderts betonten die Aktivistinnen, daß Frauen einen ganz anderen Blick auf die alltäglichen Bedürfnisse in den Familien hätten. Sie könnten schneller, umfassender und sorgfältiger sehen, woran es den Armen in der Gemeinde wirklich fehle. Ihre Kenntnis der Hauswirtschaft sei beste Voraussetzung für soziale Arbeit, eine Voraussetzung, an der es den "Gemeindevätern" ebenso wie männlichen Armenpflegern mangelte. ${ }^{18}$

Spätestens seit den 1890er Jahren sprachen Feministinnen international von „organized motherhood" bzw. „organisierter Mütterlichkeit", um die Notwendigkeit ihres Handelns zu betonen. Unter "geistiger Mütterlichkeit" verstanden sie den Einsatz für Menschlichkeit und Gerechtigkeit in einer einseitig „zweckrational“ ausgerichteten Welt, eine Art weibliche Kapitalismuskritik. Ihre soziale Praxis, die sie ebenfalls international - emphatisch "soziale Arbeit" nannten, sollte sowohl im Einzelfall "Hilfe zur Selbsthilfe" für die Betroffenen als auch der erste Schritt zu notwendigen Sozialreformen sein, welche die lebensfeindlichen Folgen der Industrialisierung aufheben würden. Die einseitig männlich geprägten modernen Gesellschaftsstrukturen wollten sie durch das Wirksam-Werden von "weiblicher Kultur" (re)humanisieren.

Soziale Arbeit umfaßte damals weit mehr als das, was heute unter "Sozialarbeit" verstanden wird. Beispielsweise gründeten die Frauenrechtlerinnen in Bremen wie in Zürich - nach englischem Vorbild - in den 1890er Jahren mit großem Erfolg alkoholfreie Speisehäuser und

$16 \mathrm{Vgl}$. Marie Elisabeth Lüders, Fürchte dich nicht. Persönliches und Politisches aus fast 80 Jahren, 1887-1962, Köln/Opladen 1963.

$17 \mathrm{Vgl}$. u. a. Maya I. Fassmann, Die Mutter der Volksküchen - Lina Morgenstern und die jüdische Wohltätigkeit, in: Christiane Eifert u. Susanne Rouette Hg., Unter allen Umständen. Frauengeschichte( $(n)$ in Berlin, Berlin 1986, 34-59.

$18 \mathrm{Vgl}$. u. a. Susanna Dammer, Mütterlichkeit und Frauendienstpflicht. Versuche der Vergesellschaftung .weiblicher Fähigkeiten" durch eine Dienstverpflichtung (Deutschland 1890-1919), Weinheim 1988. 
setzten sich nach der Jahrhundertwende und während des Ersten Weltkriegs erfolgreich für Schrebergärten und Werkskantinen ein.

Für die Begründerin der Berliner Sozialen Frauenschule, Alice Salomon (1872-1948), ${ }^{19}$ begann die außerhalb der Behörden entstehende soziale Frauenarbeit zunächst typischerweise mit der Krankenpflege. Denn leicht konnte die Unfähigkeit zum Erwerb oder zur Beaufsichtigung der Kinder während einer Krankheit eine Notlage herbeiführen, die zeitweilige Armut oder dauernde Verelendung nach sich ziehen konnte. "Hauspflegevereine" kümmerten sich seit den 1830er Jahren tatkräftig um kranke Hausfrauen und ihre Hausstände. Noch 100 Jahre später leisteten sie in der Wochenfürsorge unersetzliche Arbeit. In Bremen regten Mitglieder des Frauen-Erwerbs-Vereins bereits 1869 die Ausbildung weltlicher Krankenschwestern als neuen Ausbildungsberuf für Frauen an. Sie hofften, daß die Versorgung Verunglückter im Rote-Kreuz-Krankenhaus insbesondere den unbemittelteren Volksklassen zugute kommen werde.

In Berlin gründeten Frauen 1884 das Pestalozzi-Fröbel-Haus, um Kindergärtnerinnen und Hauswirtschaftslehrerinnen auszubilden. Für die Frauen der Frauenbewegung bestand die Grundausbildung des "allseitig gebildeten Menschen" u. a. aus der „Hauswirtschaftsschule", und zwar für Frauen aller Schichten gleichermaßen, einschließlich der sozial aktiven Frauen. Die ab 1901 von den Bremer Volksschullehrerinnen veranstalteten „Fortbildungsabende für schulentlassene Mädchen der unteren Kreise" boten den Mädchen ebenfalls insbesondere Hausarbeitswissen als "lebensweltliches Wissen" an. ${ }^{20}$ Auch die Lebensreformbewegung und der sozialhygienische Diskurs ließen zu dieser Zeit die gesellschaftliche Bedeutung des Hausarbeitswissens deutlich werden. Die durch derartige Initiativen mitangeregten später entstehenden Berufsschulen setzten die allgemeinbildende Intention dieser frühen Initiativen kaum in die Praxis um.

1884 riefen Frauen des Bremer Frauen-Erwerbs-Vereins in Verbund mit Frauenvereinen anderer Städte eine Initiative "Mädchenschutz" ins Leben, um arbeitssuchenden fremden jungen Mädchen zu bezahlbarer Unterkunft und einer Anstellung zu verhelfen. Aus Initiativen dieser Art entstanden später die Bahnhofsmissionen. Ab 1900 bemühten sich die Bremerinnen um die Resozialisierung junger, in den geschlossenen Abteilungen der Krankenanstalten festgehaltener "gefallener" Mädchen. Gleichzeitig richteten sie in präventiver Absicht Kinderhorte ein, um Mädchen, deren Eltern vollerwerbstätig waren, Aufsicht und Betreuung nach der Schule zukommen zu lassen. 1907 gründete eine kirchliche Frauengruppe in Bremen eine "Zufluchtsstätte" für obdachlose Dienstmädchen und Prostituierte. Ein Jahr zuvor hatten Bremerinnen ein „Mütter- und Säuglingsheim" für ledige Mütter gegründet. Damit sollte ein erster Schritt getan werden,

19 Zu Alice Salomon vgl. u. a. Inge Buck, Ferninistische Ansätze in der Sozialarbeit, in: Neue Ansätze in der Frauenforschung, hg. von der Wissenschaftliche Einheit Fravenstudien und Frauenforschung an der Hochschule Bremen. Bremen 1988, 150-168.

$20 \mathrm{Vgl}$. Detlev J. K. Peukert, Grenzen der Sożialdisziplinierung. Aufstieg und Krise der deutschen Jugendfürsorge von 1878 bis 1932. Köln 1986. 1071 
sowohl die elende Situation der unehelichen Mütter zu verbessern als auch die Überlebenschancen der unehelichen Kinder zu erhöhen, von denen bis dahin über die Hälfte wegen ausbleibender Alimente und unzureichenden "Haltekinder-Wesens" das erste Lebensjahr nicht überlebte. ${ }^{21}$

\section{Vernetzung und Professionalisierung der sozialen Arbeit: Auskunftsstellen und Soziale Frauenschulen}

1897 richteten die Mitglieder des Bremer Frauen-Erwerbs-Vereins nach Berliner Vorbild eine Auskunftsstelle für Wohltätigkeit ein. Sie solte sozial interessierten Frauen das richtige Projekt zur Mitarbeit vermitteln und Hilfsbedürftigen im Dschungel der unübersichtlichen Hilfsangebote zahlreicher Vereine ihrer Stadt das für sie richtige herausfinden helfen. Schließlich sollte durch Koordination der Arbeit vermieden werden, daß einige Hilfesuchende mehrfach Leistungen in Anspruch nehmen konnten, während andere leer ausgingen. Diese Auskunftsstellen - später Zentralstellen für Wohlfahrtspflege genannt - entstanden in zahlreichen Städten international zeitgleich. Sie können als Vorläufer der Zusammenschlüsse der freien Wohlfahrtsverbände gelten. Die erste Aktivität war fast überall ein „Auskunftsbuch".

Im Zuge ihrer Auskunftsarbeit wurde den Frauen klar, daß für zahlreiche Gruppen von Hilfsbedürtigen keinerlei Unterstützungen existierten. Daher sahen sie sich gezwungen, ihre Vermittlungsarbeit durch direkte Hilfsangebote zu ergänzen. In Bremen stellten die Frauen der Auskunftsstelle 1901 fest, daß es keine gesetzlich geregelte Versorgung für die Witwen gefallener Soldaten gab. Sie gründeten daher eine "Abteilung Frauenarbeit", in der sie die Soldatenwitwen mit Näharbeiten versorgten. Außerdem erschien die Gründung einer Arbeitsvermittlung sinnvoll. Ein Arbeitsamt gab es noch nicht. Private Makler hatten sich oft als Betrüger herausgestellt. - In einer „Schreibstube für Stellenlose" wurden entlassene männliche Strafgefangene in der ersten Zeit nach der Entlassung beschäftigt.

Als weiterer Schwerpunkt sozialer Arbeit hatte sich die Gesundheitsfürsorge entwickelt, die sich seit dem ersten Jahrzehnt des 20. Jahrhunderts zu einer regelrechten „Fürsorgebewegung" auswuchs. Brennpunkte waren die "Volksseuche" Tuberkulose, die hohe Säuglingssterblichkeit - jeder fünfte bis sechste Säugling starb im ersten Lebensjahr - sowie die Sorge für unterernährte, krankheitsanfällige und verkrüppelte Schulkinder. ${ }^{22}$

$21 \mathrm{Vgl}$. Meyer-Renschhausen, Kultur, wie Anm. 4, $320 f$.

$22 \mathrm{Vgl}$. u. a. Reinhard Spree, Soziale Ungleichheit vor Krankheit und Tod. Zur Sozialgeschichte des Gesundheitsbereichs im Deutschen Kaiserreich, Göttingen 1981; Sigrid Stöckel, Die Bekämpfung der Säuglingssterblichkeit im Spannungsfeld von Sozialer Hygiene und Eugenik am Beispiel Berlins im Kaiserreich und in der Weimarer Republik, Diss. Berlin 1992; Gustav Mosse u. Max Tugendreich Hg., Krankheit und soziale Lage, München 1913. 
„Berufspersonen“ und Privatleute, Vereine und Verbände, Kommunen und Krankenkassen ergriffen Initiativen zur Gründung, Leitung und zum Betrieb von Fürsorgestellen; und nach dem zeitgenössischen Urteil eines Medizinalbeamten waren es „nicht zum wenigsten Frauen, die durchaus Geschick, Verständnis und Eifer in sozialhygienischen Fragen bewiesen"23.

Diese Entwicklung inspirierte und begleitete die Neuorganisation des Fürsorgewesens auf behördlicher Ebene. Eine erste Zentralstelle für Jugendfürsorge arbeitete seit 1904 in Berlin unter Leitung Frieda Duensings mit zahlreichen ehrenamtlichen Helferinnen ${ }^{24}$ Bereits kurz vor dem Ersten Weltkrieg entstanden einige kommunale Jugendämter. Auf Reichsebene setzten die weiblichen Abgeordneten aller Parteien die Verhandlungen und Verabschiedung des Jugendwohlfahrtsgesetzes von 1922 durch, das die bisher aufgesplitterten Zuständigkeiten und Aufgaben der Jugend- und Erziehungsfürsorge vereinigte und Landesjugendämter sowie Jugendämter in jedem Kreis schuf. ${ }^{25}$ Nach Krieg und Revolution wurde die alte diskriminierende Armenpflege durch eine der Intention nach nicht mehr "ehrenrührige", präventive "Wohlfahrtspflege" ersetzt. In vielen Städten entstanden Wohlfahrtsämter. Maßgeblich daran beteiligt waren fast überall Frauen der Frauenbewegung, seit dem Ersten Weltkrieg auch Frauen der linken Parteien. Im "Innendienst" waren allerdings vielerorts die Beamten der ehemaligen Armenverwaltung weiterhin tätig, die sich lange gegen die Einstellung von Frauen gesträubt hatten. Schließlich wurde 1924 das Unterstützungswohnsitzgesetz durch die Reichsfürsorgeverordnung abgelöst. Die Ausführungsbestimmungen blieben Ländersache, die konkrete Ausgestaltung der Wohlfahrtsämter war von Ort zu Ort unterschiedlich.

In den großen Städten besonders der industriellen Ballungsgebiete wurden neben privater Vereinstätigkeit bereits früh kommunale Fürsorgeärzte und geschulte Pflegerinnen für die Aufgaben der sich schnell entfaltenden Zweige der Gesundheitsfürsorge eingestellt. Dies führte hier später zur Gründung von Gesundheitsämtern oder Gesundheitsabteilungen bei den Wohlfahrtsämtern. In kleinen Städten und auf dem Land ging die Initiative für Beratungsstunden in der Tuberkulose- und Säuglingsfürsorge neben den Kreisärzten auch von Frauenvereinen und Kreisfürsorgerinnen aus - sofern sich Aktivitäten auf diesem Gebiet überhaupt durchsetzen ließen. ${ }^{26}$

Die Vielzahl der häufig unabhängig voneinander arbeitenden sozialen Initiativen hatte zu einem unkoordinierten Nebeneinander ge-

23 Kühnlein, Die preussischen Kreisärzte und der I. Deutsche Gesundheitsfürsorgetag in Berlin am 25. Juni 1921, in: Zeitschrift für Medizinalbeamte, 34 (1921), 306-315, 336-345, hier 313

24 Elisabeth Meyer-Renschhausen, Duensing. Frieda, in: Rudolph Bauer Hg., Lexikon des Sozial- und Gesundheitswesens. A-F, München/Wien 1992, 466f.

$25 \mathrm{Vgl}$. Stoehr, Housework, wie Anm. 15; Claudia Koonz, Conflicting Allegiances: Political Ideology and Women Legislators in Weimar Germany, in: Signs, 1, 3 (1976), 663-683

26 Vgl. Kühnlein, Kreisärzte, wie Anm. 23, 309; Marie Baum, Über Säuglingsfürsorge auf dem Lande, Sonderdruck der Zeitschrift für Säuglingsschutz, 3 (1911), 1-16. 
führt. Es war geeignet, die Arbeit einzelner Helferinnen in einer Familie völlig zu untergraben, da sie von verschiedenen Seiten gleichzeitig „überrannt" wurde. „Wenn nu noch eene Schwester kommt, häng ick mir uff"27, wurde die geplagte Klientel von einsichtigen Zeitgenossen wieder und wieder zitiert. Vielfach gaben die Fürsorgerinnen verschiedener Stellen sich nicht nur die Klinke der Wohnungstür in die Hand, sondern ihrer Klientel auch einander widersprechende Ratschläge und Aufträge. Diese Mißstände führten zur Entwicklung des neuen Konzepts einer einheitlichen "Familienfürsorge".

$\mathrm{Zu}$ ersten Zentren der Familienfürsorge wurden der Verein für Säuglingsfürsorge im Regierungsbezirk Düsseldorf mit seiner Geschäftsführerin Marie Baum (1874-1964) ${ }^{28}$ seit 1907, die Wohnungsinspektion des Kreises Worms, unter der Leitung von Marie Kröhne seit 1908, und die Schulkinderfürsorge des 1894 gegründeten Charlottenburger Vereins Jugendheim mit seiner Vorsitzenden Anna von Gierke (1874-1943). Hier wurde 1909 in Verbindung mit dem Schulamt die erste "Schulhelferin" als Familienfürsorgerin eingestellt. ${ }^{29}$ Der Verein für Säuglingsfürsorgewar 1905 als gesundheitsfürsorgerischer Zweckverband sämtlicher Stadt- und Landkreise des Regierungsbezirks Düsseldorf gegründet worden. Vorstand war der Pädiater und Sozialhygieniker Arthur Schloßmann (1867-1932). Durch Auswahl, Einstellung und "ständige Fühlungnahme" mit den Fürsorgerinnen war der Verein, besonders seine Geschäftsführerin Marie Baum, eng mit der kommunalen Gesundheitsfürsorge verknüpft. Doch hatte er den Vorteil, weitgehend unabhängig von behördlichen Arbeitsweisen und onne Verbindung zur Armenpflege agieren zu können. Das Charlottenburger Jugendheim war eine Gründung der Frauenbewegung; seine Aktivitäten konzentrierten sich anfangs auf die Betreuung und Versorgung von tagsüber familienlosen Kindern in Kindergärten und -horten, auf Schulkinderspeisungen und Erholungsfürsorge. Die Wohnungsinspektion des Kreises Worms hingegen war eine kommunale Einrichtung und zielte auf die Verbesserung der Wohnqualität durch Förderung des Wohnungsbaus und der Wohnungspflege. Das Konzept der Familienfürsorge ging also von ganz unterschiedlichen Ansatzpunkten sozialer Arbeit und Notlagen aus.

Es war kein Zufall, daß es Frauen waren, die die Familienfürsorge „erfanden". Sie hatten in diesen Institutionen nicht nur leitende Positionen inne. Ihr Denken war familiengerichteter und "einheitlicher". Ihnen waren „die arbeitsteiligen Betriebsformen noch nicht so in Fleisch und Blut übergegangen wie dem Mann, den sein Beruf seit langem dazu er(zog) ${ }^{\text {" }}$ " An eine Stelle sollten Hilfesuchende sich wenden können, und von einer Stelle aus - mit Blick auf die „Einzelnot” im Familienzusammenhang - sollte der "soziale Heilplan" erarbeitet werden. Alle in Frage kommenden Aspekte waren dabei zu berück-

27 Adolf Gottstein, Das Heilwesen der Gegenwart. Gesundheitslehre und Gesundheitspolitik, Berlin 1924, 465.

$28 \mathrm{Vgl}$. Mariene Ellerkamp, Baum, Marie, in: Bauer, Lexikon, wie Anm. 24, 243.

29 Marie Baum, Familienfürsorge $(=$ Schriften des Deutschen Vereins für öffentliche und private Fürsorge), Karlsruhe '1927, ${ }^{2} 1928,6-17$.

30 Baum, Familienfürsorge, wie Anm. 29, 6. 
sichtigen, seien sie wirtschafts-, wohnungs-, gesundheits- oder jugendfürsorgerischer Art. Ziel war die "Stärkung der in der Familie liegenden Pflege- und Erziehungskräfte", also primär die Stärkung der Familienmütter. Dabei war sozial arbeitenden Frauen sehr wohl bewußt, daß Wohlfahrtspflege ohnmächtig war, wenn sie sich nicht auf die Hilfsbereitschaft von Verwandten, vor allem aber auf die Einsatzbereitschaft und Verantwortlichkeit der Mütter selbst stützen konnte. ${ }^{31}$

Während des Ersten Weltkriegs wurde die Familienfürsorge in der Arbeit des Nationalen Frauendienstes in breitem Umfang erprobt, noch weitgehend ohne Anstellung der sozialen Arbeiterinnen bei den Kommunen. In der Weimarer Zeit führten immer mehr Gemeinden bzw. Gemeindeverbände die Familienfürsorge als Organisationsprinzip und Arbeitsmethode der Wohlfahrtspflege ein. Für bestimmte abgegrenzte räumliche Gebiete („Bezirke“) wurden Bezirksfamilienfürsorgerinnen angestellt. Voraussetzung dafür war deren systematische Ausbildung mit staatlich anerkannter Abschlußprüfung. Die entsprechenden Kurse und Schulen waren seit 1893 entstanden.

Damals hatten die Frauen in ihrer "sozialen Hilfsarbeit" erfahren, daß auch die traditionelle Praxis der privaten Armenpflege grundsätzlich unzureichend war. Die alten patriarchalen Formen des bloßen "Wohltuns" versetzten die Hilfsbedürftigen in einen inferioren Status. Die Frauen wollten nun - ohne damit die Abschaffung materieller Hilfeleistungen anzustreben - dazu beitragen, die Bedürftigen durch persönliche Betreuung und Rat wieder selbständig zu machen. Wie alle Bürger des liberalen 19. Jahrhunderts glaubten sie, daß nur "Hilfe zur Selbsthilfe" nützen könne. Diese könne jedoch nur geleistet werden, wenn die Helferinnen nicht völlig unvorbereitet an die Arbeit und zu den armen Familien gingen, wie es bisher der Fall war. Die Frauenbewegten richteten daher für ihre freiwilligen Helferinnen Vortragsreihen ein, die sie auf die soziale Arbeit vorbereiten sollten.

In Berlin begannen Jeanette Schwerin (1852-1899) ${ }^{32}$ und ihre Mitarbeiterinnen ab 1893 Vortragsreihen für die "Mädchen- und Frauengruppen für soziale Hilfsarbeit" zu organisieren. Die Vorträge übernahmen Vertreter der entstehenden Soziologie, vielfach Wissenschaftler oder Sozialpolitiker des 1872 gegründeten Vereins für Sozialpolitik, die „Kathedersozialisten" und ein "social“ interessierter Mediziner. ${ }^{33}$ Später erteilten die Frauen ihren Helferinnen Unterricht in Jahreskursen. Diese Kurse sind als der Beginn einer ersten systematischen Ausbildung von künttigen Sozialarbeiterinnen zu verstehen. Gleichzeitig entstanden derartige Kurse in anderen Ländern. Alice Salomon, Nachfolgerin der früh gestorbenen Jeanette Schwerin, bezog sich auf diese Gründungen, als sie zusammen mit anderen

31 Zu Aufgabe. Ziel, Methode und Form der Familienfürsorge vgl. Baum, Familienfürsorge, wie Anm. 29, 32-53

$32 \mathrm{Vgl}$. Elisabeth Meyer-Renschhausen, Schwerin, Jeanette, in: Bauer, Lexikon, wie Anm. 24, P-Z, 17024 .

$33 \mathrm{Vgl}$. Elisabeth Meyer-Renschhausen, Soziologie, soziale Arbeit und Frauenbewegung. Eine Art Familiengeschichte, in: Feministische Studien, 12, 1(1994). 17-32; vgl. auch Sachße, Mütterlichkeit, wie Anm. 7. 1161. 
Frauen 1908 in Berlin die erste reguläre Soziale Frauenschule aus der Taufe hob. ${ }^{34}$

Im Verlauf des Ersten Weltkriegs wurde es in zahlreichen Kommunen klar, daß sie auf die Arbeit ausgebildeter Sozialarbeiterinnen angewiesen waren. Jetzt wurde allgemein akzeptiert, was die Frauenbewegung schon lange gefordert hatte, nämlich daß eine schulmäßige Vorbildung der Armenpfleger/innen und aller sonst "sozial" Arbeitenden unabdingbar war. Verschiedene Stadtgemeinden entschlossen sich, die Gründung von Sozialen Frauenschulen finanziell zu unterstützen. Noch während des Kriegs und vor allem in der Nachkriegszeit entstanden Soziale Frauenschulen, vielfach unter der Leitung bekannter Frauenrechtlerinnen. ${ }^{35}$ Allein während der Jahre 1916 bis 1918 wurden 13 solche Schulen eröffnet. 1929 gab es in Deutschland 37 Wohlfahrts- bzw. Soziale Frauenschulen, fünf davon für männliche Schüler. ${ }^{36}$

Unterricht und Seminare an den Sozialen Frauenschulen waren damals zweifelsohne der Zeit und ihren reformpolitischen Diskursen gegenüber weit aufgeschlossener als dies an den Universitäten der Fall war. Der Fächerkanon an der Bremer Sozialen Frauenschule umfaßte 1920 ein differenziertes Angebot, das von Fächern wie Deutsch und Geschichte, Volkswirtschaftslehre, Sozialpolitik und Arbeitsrecht, Staats- und Rechtskunde, Verwaltungslehre, Sozialhygiene, Soziale Fürsorge und Jugendfürsorge über Psychologie und Erziehungslehre, Berufskunde, Bürotechnik, Organisationslehre bis hin zu Führungen und Besichtigungen reichte. Die Ausbildung dauerte zwei Jahre. Vorausetzung für die Aufnahme war eine abgeschlossene Schulbildung (Volksschule konnte ausreichen), eine Fachausbildung etwa als Kranken- oder Säuglingsschwester oder die Teilnahme an der Allgemeinen Frauenschule nebst einem anschließenden Jahr sozialer Praxis. Der Fächerkanon entsprach den Prüfungsanforderungen, wie sie die 1916 unter der Leitung Alice Salomons gegründete „Konferenz der Sozialen Frauenschulen Deutschlands" zunächst in Preußen 1920 hatte durchsetzen können und wie sie 1922 auch für Bremen festgelegt wurden. 1931 wurde diese zweijährige Ausbildung mit anschließendem Anerkennungsjahr in der Praxis auf Reichsebene einheitlich anerkannt. ${ }^{37}$

Eine der damals jungen Frauen, Dora Hansen-Blancke, berichtete, daß sie, als sie hörte, daß in Hamburg eine Soziale Frauenschule mit führenden Frauen aus der Frauenbewegung eröffnet werden würde, sofort beschloß, dort hinzugehen. Das Zentrum des Neuen und des Lebens lag für sie in der Sozialen Frauenschule - im Gegensatz zu

$34 \mathrm{Vgl}$. Wolf Rainer Wendt, Geschichte der sozialen Arbeit, Stuttgart ${ }^{2} 1985,166 f$. 35 So in München Frieda Duensing, in Hamburg Marie Baum und Gertrud Bäumer

36 Alice Salomon, Offentliche und freie Wohlfahrtspflege, in: Bernhard Möllers Hg. Gesundheitswesen und Wohlfahrtspflege im Deutschen Reiche, Berlin/Wien '1924, ${ }^{2} 1930,576-607,610$.

$37 \mathrm{Vgl}$. Young Sun Hong. Feminity as a Vocation: Gender and Class Conflict in the Professionalization of German Social Work, in: Geoffrey Cocks $u$. Konrad $H$. Jarausch Hg., German Prolessions 1800-1950, New York/Oxford 1990, 232-251. hier $238 f$. 
den ihr verstaubt erscheinenden Universitäten. Die Mädchen waren begeistert vom Unterricht, ihren Lehrerinnen und "der Arbeit". Sie wohnten entsprechend den Idealen der Settlement-Bewegung in den Arbeitervierteln. Gemeinsam mit Betroffenen managte die junge Dora Hansen-Blancke mit Erfolg ein Obdachlosen-Asyl. ${ }^{38}$ Das Lernen und Arbeiten in den Seminaren wurde von Schülerinnen und Lehrerinnen wie gelebte soziale Bewegung empfunden. Die Lehrerinnen hielten Kontakt zur Frauenbewegung, deren Tagungen sie regelmäßig besuchten. ${ }^{39}$ Auch aus anderen Berichten der "Pionierzeit der Sozialarbeit" wissen wir, daß Schülerinnen und junge Sozialarbeiterinnen damals Sozialarbeit als Kern der Arbeit für "eine neue Zeit" begriffen. ${ }^{40}$

Nach der Machtübernahme durch die Nationalsozialisten 1933 wurde die demokratische Leiterin der Allgemeinen Sozialen Frauenschule in Bremen entlassen, die Schule verstaatlicht und in Staatliche Fachschulen für Frauenberufe umbenannt. Weil die Nationalsozialisten jedoch kaum auf eigene Fachkräfte zurückgreifen konnten, mußten sie doch wieder eine selbstbewußte Reformerin, Elisabeth Siegel, als Schulleiterin einsetzen. Der Frauen-Erwerbs-Verein als Trägerverein versuchte, durch Kompromisse zu überwintern. Er behielt zwar Grundstücke und Häuser, stellte aber ein Haus der nationalsozialistischen Frauenschaft zur Verfügung und ließ $z u$, daß es in „Karin-Göring-Haus" umbenannt wurde. Gegen Ende des Zweiten Weltkriegs wurden die Gebäude zerbombt. Nach Kriegsende wurden zwar im Oktober 1945 im Keller eines Hauses Kurse und Mittagstische wieder eröffnet, ${ }^{41}$ aber später entstand auf den ehemaligen Grundstücken der Sozialen Frauenschule ein Berufsschulzentrum. Um 1950 wurde die ehemalige Bremer Soziale Frauenschule auch für männliche Schüler geöffnet. Erst die Bildungsreform ab 1970 führte zu einem männlich dominierten Lehrkörper. Die Angliederung der Hochschule für Sozialarbeit und Sozialpädagogik (HfSS), wie die Einrichtung später hieß, an die Bremer Hochschule hat die weiblichen Spuren vollends verwischt.

\section{Zwischen Medikalisierung und Bürokratie: Kontroversen um die Familienfürsorge}

Die Fachgeschichte tut sich mit den „weiblichen“ Wurzeln der Sozialarbeit schwer. Der "Paradigmen-Wechsel" der Zeit nach 1968 erlaubte keinen positiven Zugang zu ihrer "mütterlichen" Herkunft.

38 Gespräch und Interview Elisabeth Meyer-Renschhausen mit Dora Hansen-Blancke am 1.6.1980 und 13.1.1983 in Hamburg.

39 Staatsarchiv Bremen (StAB), 3 - V.2. No 101218.

40 Interview Elisabeth Meyer-Renschhausen mit Prof. Elisabeth Siegel am 8.10.1985 in Osnabrück; vgl. auch Sabine Hering u. Edith Kramer Hg., Aus der Pionierzeit der Sozialarbeit. Elf Frauen berichten, Weinheim/Basel 1984, insbes. 23f; Elisabeth Siegel, Dafür und Dagegen. Ein Leben für Sozialpädagogik, Stuttgart 1981; Gudrun Pausewang, Rosinkawiese, München 1980.

41 Weser-Kurier (14.3.1967). 
Sozialarbeit sollte mit den „Frauenrollen" der modernen Gesellschaft nichts zu tun haben..$^{42}$ In ihrer vielzitierten Untersuchung konzedieren Christoph Sachße und Florian Tennstedt zwar, daß „bedeutsame Anstöße für die Entwicklung kommunaler Fürsorge ... aus der bürgerlichen Frauenbewegung (kamen)"43 die Darstellung der sozialen Frauenarbeit jedoch bleibt recht blaß. Dies beruht auf der Vorrangstellung, die die Autoren den zeitgenössischen Wissenschaften und den Ärzten bei der Entstehung und Ausprägung der modernen Wohlfahrts- und Gesundheitspflege einräumen. Ohne Zweifel hatten die Soziologie und vor allem die Sozialhygiene als "Leitwissenschaften" auf die Gesundheitsfürsorge großen Einfluß. ${ }^{44}$ Nun wollten die Frauen inre Schulen keineswegs als „unwissenschaftlich" verstanden wissen; auch waren sie in der Gesundheitsfürsorge nicht nur marginal beteiligt. Ihre Arbeit war vielmehr unerläßlich. „Für das Wohlfahrtsamt ist die Kreisfürsorgerin das, was die Welt im innersten zusammen hält"45, heißt es in einem Standardwerk der Sozialhygiene aus der Weimarer Zeit. Diese Wertschätzung der fürsorgerischen Frauenarbeit kehrt in vielen zeitgenössischen Schriften der 1920er Jahre wieder. Der Erfolg in der Gesundheitsfürsorge wurde häufig als von der Arbeit dieser "besonderen Hilfsorgane" überhaupt abhängig begriffen.

In der Gesundheitsfürsorge verband sich die junge Wissenschaft der Sozialhygiene mit der neuen, von der Frauenbewegung entwickelten sozialen Arbeit. Die Sozialhygieniker verknüpften Verfahren der Medizin, Volkswirtschaft, Soziologie und Statistik, um das Verhältnis von Krankheiten und Gesellschaft zu erforschen. Aus den so gewonnenen Erkenntnissen leiteten sie sozialpolitische Forderungen und Normen für die praktische Gesundheitsfürsorge ab. Ihr Ziel war „die Verallgemeinerung hygienischer Kultur unter der Gesamtheit von örtlich, zeitlich und gesellschaftlich zusammengehörigen Individuen und deren Nachkommen"46, wie es Alfred Grotjahn, 1920 der erste Lehrstuhlinhaber dieses Faches, im Jahr 1904 definierte.

Orte der Gesundheitsfürsorge und historisches Novum waren die „Fürsorgestellen", in denen Fürsorgerin und Arzt (oder Ärztin) gemeinsam tätig waren. Ärztliche Sprechstunde und Außenfürsorge der Sozialen Arbeiterin in Wohnung und Familie waren dabei eng miteinander verbunden. „Viele Krankheitszustände bedürfen keiner (gewöhnlichen) Rezeptvorschrift, sondern nur einer vernünftigen Pflege,

42 Vgl. hierzu kritisch Hans Drake, Frauen in der Sozialarbeit. Sexismus und geschlechtsspezifische Diskriminierung. Neuwied 1980.

43 Sachße/Tennstedt. Geschichte, wie Anm. 15, 42, Beispiele: 30f, 119, $128 \mathrm{f}$

$44 \mathrm{SachBe} / T e n n s t e d t$, Geschichte, wie Anm. 15, 18-23; Alfons Labisch u. Florian Tennstedt, Der Weg zum „Gesetz zur Vereinheitlichung des Gesundheitswesens" vom 3. Juli 1934. Entwicklungslinien und -momente des staatlichen und kommunalen Gesundheitswesens in Deutschland, Düsseldorf 1985; Meyer-Renschhausen, Soziologie, wie Anm. 33.

45 Peter Krautwig. Die Organisation der Gesundheitsfürsorge, insbesondere die Aufgabe von Provinz, Stadt- und Landkreisen auf dem Gebiet der Gesundheitsfürsorge, in: Adolf Gottstein u. a. Hg. Handbuch der Sozialen Hygiene und Gesundheitsfürsorge, I: Grundlagen und Methoden, Berlin 1925, 439-495, hier 464f.

46 Alfred Grotjahn. Was ist und wozu treiben wir soziale Hygiene? in: Beilage zur Hygienischen Rundschau, 14 (1904), 1017-1032, hier 1026. 
geeigneter Diät, eines ganz allgemein hygienischen oder erzieherischen Rates" 47 , war das gesundheits- und sozialpolitische Credo der in der Gesundheitsfürsorge Tätigen. Damit ist zugleich das Besondere und Neuartige ihrer Arbeit gekennzeichnet. Anders als sonst bei ärztlichen Konsultationen wurde in den Fürsorgestellen nicht behandelt, sondern untersucht, beraten und „aufgeklärt". Das „,sozialhygienische Rezept" basierte auf dem ärztlichen Körperbefund und der "sozialen Diagnose", die auf der Beurteilung der Verhältnisse des Betroffenen und seiner Familie seitens der Fürsorgerin beruhte. Es „verschrieb" jeweils konkrete Empfehlungen hinsichtlich Ernährung, Wohnung, (Haus-)Wirtschaft. Daneben konnten Überweisungen zur ärztlichen Behandlung, die Einleitung von Erholungskuren, Speisungen und Hilfestellung bei Anträgen auf Zuschüsse von Krankenkassen stehen.

Ohne die ärztliche Fachkompetenz in gesundheitlichen Fragen anzuzweifein, hatten die sozial arbeitenden Frauen eine andere Perspektive als die Ärzte. Marie Baum betonte stets die „Unteilbarkeit von Leib und Seele" im sozialen wie im kulturellen Familienzusammenhang als Grundlage der Sozialarbeit und lehnte „Fürsorge aus einer rein auf die körperliche Gesundheit eingestellten Betrachtungsweise" ab. ${ }^{48}$ In dem von ihr herausgegebenen "Grundriß der Gesundheitsfürsorge" beschrieb sie als "Sinn und Zweck der offenen Fürsorge", "durch Rat und Belehrung in mühsamster Kleinarbeit die zur Erhaltung und Pflege der Volksgesundheit erforderlichen Kenntnisse zu verbreiten, auf wahrem Vertrauensverhältnis fußend Einsicht und Kräfte derer zu stärken, die Hüterinnen der Volksgesundheit sind - der Mütter -, und im Verein mit der wirtschaftlichen und Erziehungshilfe einer für jeden Einzelfall gut durchdachten Art vorgefundenen Nöten zu steuern, die der Gesundheit des einzelnen hindernd im Wege stehen " 49.

Wenn auch die Arbeitsteilung zwischen ärztlicher und fürsorgerischer Tätigkeit „selbstverständlich“ war, war sie dennoch häufig konfliktreich. Die Konflikte können als Erschütterung des traditionell-bürgerlichen Weltbildes von männlicher Berufs- und Geschlechtsidentität verstanden werden. Obwohl der fürsorgeärztliche Beruf in der Weimarer Zeit nicht mehr so eng an das Geschlecht gebunden war wie der Beruf der Fürsorgerin, ${ }^{50}$ wurde er in den sozialhygienischen Diskursen doch vornehmlich „männlich“ konnotiert. Diese Diskurse schrieben den Sozialmedizinern das Wissen bzw. die Erkenntnis, den Fürsorgerinnen die Praxis mit den dazu nötigen Kenntnissen

47 P. Stephani, Fürsorge für das schulpflichtige Alter, in: Möllers, Gesundheitswesen, wie Anm. 36, 230-243, hier 236

48 Baum, Familienfürsorge, wie Anm. 29, 123.

49 Marie Baum Hg. Grundriß der Gesundheitsfürsorge, '1919, ${ }^{2} 1923$, XXI.

50 So nahm die Zahl der "weiblichen Ärzte" in der Gesundheitsfürsorge $z u$, während die ersten männlichen Sozialarbeiter, aus der Jugendbewegung kommend, nicht im Bereich der Gesundheits- und Familienfürsorge, sondern der Jugendpflege tătig waren; vgl. Beate Ziegeler, Weibliche Ärzte und Krankenkassen. Antänge ärztlicher Berutstätigkeit von Frauen in Berlin 1893-1935. Weinheim 1993, 27f: Atina Grossmann. Berliner Ärztinnen und Volksgesundheit in der Weimarer Republik: Zwischen Sozialreform und Eugenik, in: Eifert/Rouette, Unter allen Umständen, wie Anm. 17, 183-217. 
zu. Diese Arbeitsteilung implizierte nur zu oft ein hierarchisches Verhältnis.

Viele Konflikte beruhten auf einem unterschiedlichen Verständnis von "Praxis". Eine häufige Kritik der Wohlfahrtspflegerinnen an den Ärzten war, daß sie von innen rein technische Hilfeleistungen in der Sprechstunde und statistische Hilfsarbeiten verlangten, die ihnen für ihre Hauptaufgabe, die Arbeit mit den Familien in der Außenfürsorge, zu wenig Zeit ließen. Der Arzt war von Klinik und Ausbildung her die ihm untergeordnete Krankenschwester gewöhnt. Die Fürsorgerin aber trat ihm als „Sozialberaterin“ gegenüber. Bei der Erstellung der „Sozialen Diagnose" war er auf ihre Mitarbeit angewiesen. Es fiel vielen Ärzten schwer, die Fürsorgerin als Fachkraft zu akzeptieren. Stattdessen befürchteten sie, „daß die Fürsorgerin dem Arzt ins Handwerk pfusche" ${ }^{1}$. Aus Sicht der weitgehend selbständig arbeitenden Bezirksfürsorgerinnen begannen die Probleme häufig jedoch erst dann, wenn Stadt oder Kreis einen Fürsorgearzt einstellte, also einen Schritt in Richtung „Medikalisierung" der Gesundheitsfürsorge tat.

Ursachen dieser Auseinandersetzungen werden im Streit um die Ausbildung deutlich, der über Fragen der hierarchischen Arbeitsteilung weit hinausging. Die in vielen Variationen vorgebrachte Kritik, „die Zöglinge der sozialen Schulen (seien) zwar sehr gelehrt, aber praktisch doch oft nicht hinreichend geschult" 52 , mag in Einzelfällen zutreffend gewesen sein. Typischer war aber die vor allem von ärztlicher Seite vorgetragene Auffassung, die Ausbildung an den Sozialen Frauenschulen sei zu umfassend - gerade darauf legten jedoch letztere besonderen Wert. ${ }^{53}$ Ein ärztlicher Befürworter der guten Ausbildung von Fürsorgerinnen interpretierte die „heftigen Kontroversen" um die Ausbildung und die "wechselnden gesetzgeberischen Akte" Mitte der 1920er Jahre offen als Geschlechterkonflikt: Die Heranziehung der Frau als "Hilfsorgan der Gesundheitsfürsorge" habe zu einer "gewissen Vermännlichung" ihrer früher mehr "sentimentalen", subjektiven Einstellung in der Wohlfahrtsarbeit geführt. Die moderne Gesundheitsfürsorgerin, wie die Wohlfahrtspflegerin überhaupt, verkörperte für inn einen weiblich-männlichen „Dualismus". Im Streit um die Ausbildung sei die eine Richtung bestrebt, aus der Frau, die "durch Mitleid wissend war und ist, eine neue Frau zu gestalten, die aus Wissen mitleidig werde". Das ging eindeutig an die Adresse der Frauenbewegung. Die „andere Richtung" wolle, auf der „praktischen Betätigung" aufbauend, die Frau als Gesundheitsfürsorgerin "lediglich mit dem geistigen Rüstzeug ausstatten, das sie in ihrer Tagesarbeit nicht entbehren könne - sie bewahrend vor einer Gelehrsamkeit, die dazu führe, daß , man den eigenen Vater nicht mehr kennt" "54.

51 Stephan Engel u. Hans Meyer, Fürsorge für Mütter, Säuglinge und Kleinkinder, in: Möllers, Gesundheitswesen, wie Anm. 36, 208-230, hier 219.

52 Engel/Meyer, Fürsorge, wie Anm. 51, 215.

53 Versuche des preußischen Innenministeriums, eine Kurzausbildung speziell für Gesundheitsfürsorgerinnen durchzubringen, scheiterten; vgl. Hong, Feminity, wie Anm. 37, 2388 .

$54 \mathrm{H}$. Rosenhaupt, Die Hilfsorgane der Gesundheitsfürsorge, ihr Wirkungskreis und ihre Ausbildung, in: Adolf Gottstein u. a. Hg., Handbuch der Sozialen Hygiene, IV: 
Das Interesse an einer geschlechtsspezifisch definierten klaren Berufshierarchie (männliches "höheres" Wissen versus weibliche „niedere" Praxis) vermischt sich hier - aus heutiger Sicht völlig unerwartet - mit einem „privaten" familienbezogenen Argument, dem der Solidarität mit dem Vater gegen die "gelehrte" Tochter. Hierin spiegelt sich eine Facette der sich um diese Zeit vollziehenden "Auflösung" der traditionell-bürgerlichen „Ordnung der Geschlechter", ein weiblicher "Einbruch" in die vormals rein männlich besetzte öffentliche (Berufs- und Geistes-)Welt und damit eine Umstrukturierung vormals getrennter Sphären. Dieser "Einbruch" war von der Frauenbewegung beabsichtigt, nicht aus Gründen der "Gleichberechtigung", sondern um der "Sache" willen. Für Marie Baum war die Familienfürsorge „einer der wenigen Punkte der gesellschaftlichen Entwicklung, an dem wir angefangen haben, mit dem so oft als Schlagwort ausgesprochenen Einschlag weiblicher Wesensart in das Gewebe des öffentlichen Lebens Ernst zu machen"55.

Bei der Entfaltung und Ausprägung der Fürsorge haben wir es mit einer etwa zeitgleichen Professionalisierung zweier neuer Berufsgruppen zu tun, die eng zusammenarbeiteten: jener der Fürsorgerinnen und jener der Fürsorgeärzte und -ärztinnen. Seitdem in der Weimarer Republik die moderne Wohlfahrtspflege als primär kommunale und staatliche Aufgabe formuliert und organisiert wurde, ging es für beide Gruppen um Positionsbestimmungen gegenüber der "alten" Beamtenadministration (Stadtverwaltung, Medizinalbeamte), um die Art und Weise ihres "Einbaus" in die Verwaltung und damit verbunden - um eine Neubestimmung ihres Verhältnisses.

Die Frauenbewegung hatte durchaus Erfolge zu verbuchen, war sie doch prägend an der Herausbildung des modernen Wohlfahrtsstaates beteiligt. Von sozialmedizinischer Seite wurden "Terraingewinne" der Frauen jedoch häufig als Mißachtung der ärztlichen Arbeit und Schwächung ihrer Position gewertet. So rief das Reichsjugendwohlfahrtgesetz heftige Kritik bei Sozialhygienikern, Fürsorgeärzten und Medizinalbeamten hervor, weil sie zunächst überhaupt nicht und später nicht als mögliche Leiter von Jugendämtern berücksichtigt worden waren, obwohl sie seit langem in der Säuglingsfürsorge arbeiteten. Keinesfalls bedürften sie der „Führung durch sozial geschultes Personal" - sprich Frauen - als Leiterinnen der neuen Jugendämter. ${ }^{56}$

Die von den Kommunalärzten wie den Medizinalbeamten verlangte Unterstellung der Gesundheitsfürsorge für alle Lebensalter unter ärztliche Leitung in reichsweit zu gründenden Gesundheitsämtern wurde erst im Nationalsozialismus unter rassenhygienischem Vorzeichen verwirklicht. Allerdings war ein zentralisiertes, verstaatlichtes $\mathrm{Ge}$ sundheitssystem niemals das berufspolitische Ziel der Kommunalärzte, sondern das der Medizinalbeamten. ${ }^{57}$

Gesundheitsfürsorge, soziale und private Versicherung, Berlin 1927, 678-714, hier 680. 55 Baum, Familienfürsorge, wie Anm. 29, 127.

56 Stöckel, Bekämpfung, wie Anm. 22, 237ł, zu den Auseinandersetzungen vgl. ebd. 57 Vgl. Gabriele Czarnowski, Das kontrollierte Paar. Ehe- und Sexualpolitik im Natio- 
Ein anderer Erfolg der Frauenbewegung, dem viele Fürsorgeärzte ablehnend gegenüber standen, war die Ubernahme des Konzepts der Familienfürsorge (wie reduziert auch immer) im stark expandierenden Wohlfahrtssystem der Weimarer Zeit. Die Zusammenfassung aller Fürsorgezweige unter dem Dach eines großen Wohlfahrtsamtes mit einer einheitlichen Familienfürsorgerin wurde auch von vielen Verwaltungsbeamten und vom Deutschen Verein favorisiert. Sozialmediziner sahen hier zum einen unüberwindbare organisatorische Probleme, vor allem in der Großstadt. ${ }^{58}$ Es sei „nicht einzusehen, daß man das System auf der Fürsorgerin aufbauen soll und nicht auf dem Arzt, dessen Hilfsorgan ja doch schließlich die Fürsorgerin ist oder sein soll"59. Hier ging es um Verteidigung oder Etablierung des sozialhygienischen Spezialistentums im administrativen Bereich, das durch die übergreifende Familienfürsorge gefährdet erschien.

Marie Baum, als Protagonistin der Familienfürsorge ebenso geachtet wie angegriffen, bekannte 1927: „Schwer traf mich kürzlich das Wort eines bekannten Sozialarztes, ich hätte durch meine Lebensarbeit dazu beigetragen, die Familienfürsorge der Hand des Arztes zu entwinden und an die Schreiberkaste ... auszuliefern" ${ }^{\prime 60}$. Die Familienfürsorge konnte jener Sozialarzt nur als Streitobjekt zwischen zwei männlich gedachten Berufsgruppen sehen. Demgegenüber war für Marie Baum allerdings selbstverständlich, daß die Familienfürsorge ihre administrative Verortung bei der allgemeinen Verwaltung als Träger der Wirtschaftsfürsorge verlange, weil es hauptsächlich wirtschaftliche Schäden waren, die Familien und Familienlose in Krankheit und Not stürzten - eine Auffassung, die theoretisch auch von den Sozialhygienikern vertreten wurde. Für das Verhältnis der Familienfürsorge zu den Spezialfürsorgen in Großstädten führte Marie Baum als Vergleich das Hausarzt-Facharzt-System an. Sie trat für eine weitgehende Dezentralisierung in überschaubare Fürsorgebezirke ein, die an zentraler Stelle durch eine sozial ausgebildete Akademikerin geleitet werden sollten. Die Leitung der Familienfürsorge sollte gleichrangig neben die der Armenbehörde bzw. des Wohlfahrtsamtes im engeren Sinn (der Wirtschaftsfürsorge), Jugendamt und Gesundheitsamt bzw. Fürsorgearzt, treten. Alle gemeinsam sollten einem Wohlfahrtsdezernat unterstellt sein, auf dem Land in einfacherer Besetzung dem Landrat.

Tatsächlich jedoch war diese Form der selbständigen administrativen Verankerung der Familienfürsorge, abgesehen von einigen „Oasen“, Ende der 1920er Jahre noch kaum Realität. Das Prinzip der Familienfürsorge hatte zwar gegenüber dem unkoordinierten Nebeneinander der Einzelfürsorgen in vielen Städten und Kreisen "gewonnen", blieb jedoch in der Regel auf die bloß technische Organisation des Außendienstes durch Bezirksfürsorgerinnen beschränkt. „Vorläufig fehlt es noch an der Anerkennung der Familienfürsorge als einer

nalsozialismus, Weinheim 1991, bes. Kap. 4 u. 5; Labisch/Tennstedt, Weg, wie Anm. 44 58 Krautwig. Organisation, wie Anm. 45, 470.

59 Engel/Meyer, Fürsorge, wie Anm. 51, $229 f$.

60 Baum, Familienfürsorge, wie Anm. 29, 123. 
nach Ziel und Methode unter bestimmten eigenen Gesetzen stehenden und von den sonstigen Verwaltungstätigkeiten sich unterscheidenden Berufstätigkeit, wie sie dem Lehrer, Arzt und Techniker schon längst zuteil wird"61, stellte Marie Baum in ihrer empirischen Studie 1927 fest. Sie befürchtete, daß die Familienfürsorge als Trägerin des sozialpädagogischen Einflusses auf Dauer ins Hintertreffen geraten werde gegenüber der Vertretung der gesundheitlichen und wirtschaftlichen Interessen (im Amt), wenn die Leitung der Familienfürsorge nicht gleichberechtigt neben dem Verwaltungsbeamten und dem Arzt steht. ${ }^{62}$ Ein "der Frauennatur im besonderen Maße angepaßter Beruf" sei so "in der Gefahr der Subalternisierung"63

Marie Baum kritisierte das nicht aus Gründen der beruflichen "Gleichstellung" von Männern und Frauen. Grund war die Besonderheit der fürsorgerischen Aufgabe, zwischen den Unberechenbarkeiten des individuellen (Familien-)Lebens und der "Rationalität" (Gesetzmäßigkeit) des Staates und seiner Sozialpolitik zu vermitteln. Marie Baum sprach von „beseelten Händen“, die hier eine Brücke schlagen sollten. Die Familienfürsorge war dabei durchaus kein statischer oder gar sakrosankter Komplex eines per se ideologisch besetzten Irrationalen. Die Erkenntnis der Nöte vor Ort führte dazu, bestimmte wiederkehrende Formen der Hilfe in Sozialpolitik, d. h. gesetzlich geregelte Ansprüche, umzuwandeln und so Raum und Zeit für neue Aufgaben zu schaffen.

Bevor sie sich überall hatte durchsetzen können, war die Familienfürsorge in der Krise. Dies lag vor allem daran, daß Krieg und Inflation die Aufgaben der Fürsorge nach 1914 außerordentlich vermehrt hatten, ohne daß entsprechend mehr Fürsorgerinnen eingestellt wurden. Aufgrund der Verordnung über die Fürsorgepflicht vom 13. Februar 1924 traten neben die früheren „Armen“ die Kriegsbeschädigten und Kriegshinterbliebenen, Sozial- und Kleinrentner/innen, Schwerbeschädigte und Schwererwerbsbeschränkte, hilfsbedürftige Minderjährige, schwangere Frauen und Wöchnerinnen. Hinzu kamen die durch das Reichsjugendwohlfahrtsgesetz gestellten Aufgaben und die freiwilligen Leistungen der kommunalen Gesundheitsfürsorge. Die viel zu wenigen Sozialarbeiterinnen waren völlig überlastet. Sie verfügten nicht über die finanziellen und zeitlichen Ressourcen, um wirklich helfen zu können. Marie Baum kritisierte die "Methoden des Großbetriebes" in der Wohlfahrtspflege als menschenunwürdig. Ebenso untragbar war für die Sozialarbeiterinnen ihre Unterstellung unter sozial nicht ausgebildete Beamte (häufig noch die alten Armenbeamten), die nach Verwaltungsprinzipien arbeiteten. Oft genug leiteten sie die von den Sozialbeamtinnen dringend empfohlenen Maßnahmen nicht ein, was diese häufig erst von der Klientel erfuhren.

Die Fürsorgerinnen waren in der Regel schlecht bezahlt und arbeiteten unter unsicheren Anstellungsbedingungen. Die Berufsgruppe

61 Baum, Familienfürsorge, wie Anm. 29, 125.

62 Baum, Familienfürsorge, wie Anm. 29, 60.

63 Baum. Familienfürsorge, wie Anm. 29, 119. 
war durch einen hohen Krankenstand und Frühinvalidität gekennzeichnet. Auf die Dauer, schrieb Gertrud Bäumer bereits 1924, seien „die äußeren Bedingungen, unter denen die Sożialarbeiterinnen ... arbeiten, ein Hohn auf die obligate idealistische Wertung der sozialen Arbeit" 64 .

In der Zeit des Nationalsozialismus wurden die Bezirksfürsorgerinnen den Amtsärzten als Leitern der neuen staatlichen Gesundheitsämter zu- und untergeordnet. Dies betraf insbesondere die Landkreise. Hier hörte die selbständige Familienfürsorge so gut wie ganz auf. In einigen Städten blieb sie unter mehrere „Dienstherren" aufgeteilt. $\mathrm{Ob}$ die wenigen selbständigen Leitungen der Familienfürsorge bestehen blieben, ist noch nicht erforscht. In dieser Hinsicht bedeutete der Nationalsozialismus den Sieg der Ärzte über die sozial arbeitenden Frauen und in der Tat ihre "Subalternisierung" - unabhängig davon, wie sie sonst zu dem neuen System mit seinen rassenpolitischen Zielen standen, das auch ihre Berufsarbeit neu bestimmte. ${ }^{65}$

Trotz wichtiger Vorarbeiten fehlt eine Geschichte der Sozialarbeit als Frauenarbeit in den 1920er Jahren noch immer. ${ }^{66}$ Die Art und Weise des Einbaus der "Frauenkräfte" in alte und neu entstehende Bürokratien der Weimarer Republik ist bisher noch nicht ausreichend untersucht. Ebenso mangelt es an eingehenden, auf Archivstudien gestützten Analysen des Verhältnisses zwischen sozial arbeitenden Frauen und ihrer Klientel, wie sie etwa von Susan Pennybacker für London oder von Linda Gordon für Boston vorliegen. ${ }^{67}$ Eine genaue Aufarbeitung der „anderen Geschichte“ weiblicher sozialer Reform der Weimarer Zeit wäre es wert, mit konsequentem Perspektivenwechsel in Angriff genommen zu werden - nicht zuletzt auch, um ihre Bedeutung in der Zeit des Nationalsozialismus angemessener beurteilen zu können.

64 Gertrud Bäumer, Die Stellung der Sozialbeamtin und der Sinn der Wohlfahrtspflege, in: Die Frau (1923/24), 262-265, hier 263.

$65 \mathrm{Vgl}$. Emilija Mitrovic, Fürsorgerinnen im Nationalsozialismus: Hilfe zur Aussonderung, in: Angelika Ebbinghaus $\mathrm{Hg}$., Opfer und Täterinnen. Frauenbiographien des Nationalsozialismus, Nördlingen 1987, 14-36, und andere Beiträge des Buches: Czarnowski, Paar, wie Anm. 57, 142-144, $157 \mathrm{f}$.

$66 \mathrm{Vgl}$. Susanne Zeller, Volksmütter mit staatlicher Anerkennung. Fraven im Wohlfahrtswesen der zwanziger Jahre, Düsseldorf 1987

67 Susan D. Pennybacker, Mitgefühl und Takt. Einige Widersprüche zwischen Frauen, dem Magistrat und der Sozialpolitik in London, 1889-1914, in: Dagmar Reese u. a Hg., Rationale Beziehungen? Geschlechterverhältnisse im Rationalisierungsprozeß, Frankfurt a. M. 1993, 295-318; Linda Gordon. Heroes of Their Own Lifes. The Politics and History of Family Violence, Boston 1880-1960, London 1989 